\title{
Urological Procedures in Urolithiasisand Their Association with Chronic Kidney Disease
}

\author{
Matthew D'Costa, MD; Radmila Savcic-Kos, MD; Jingbo Huang, MD; \\ Andrew D. Rule, MD; and Narayana Murali, MD
}

\begin{abstract}
Background and Objectives: Epidemiological evidence suggests that patients with urolithiasis are at increased risk for end-stage renal disease (ESRD). It is unclear if urological intervention impacts the progression of chronic kidney disease (CKD).
\end{abstract}

Methods: We conducted a retrospective observational cohort study of patients in the Marshfield Epidemiologic Study Area database between January 1991 and May 2007, where 1,340 patients diagnosed with urolithiasis were extracted. Of the I,340 subjects, 446 had urological procedures for management of urolithiasis. Those that underwent these procedures were compared to those that did not. Cox proportional hazards models adjusted for age, gender, and comorbidities were performed to evaluate the risk for CKD, elevated serum creatinine, and any-cause mortality.

Results: Baseline comorbidities in patients with and without procedures were not significantly different except for obesity $(P<0.000 \mathrm{I})$. Subjects that underwent procedures were at increased risk for elevated serum creatinine (Hazard Ratio $(\mathrm{HR})[95 \% \mathrm{Cl}]=1.49$ [I.19-1.85]) when compared to those that did not undergo a urologic procedure during the study period. The results did not reveal a significant difference in incidence of CKD or any-cause mortality.

Conclusions: Patients who undergo urologic procedures are at increased risk for elevated creatinine. Urological procedures do not appear to impact incidence of CKD or mortality and, in fact, may prevent long-term renal dysfunction.

Keywords: Chronic kidney disease; Urolithiasis; Nephrolithiasis; Urological procedures

$\mathrm{T}$ The burden of urolithiasis has been increasing, with lifetime incidences of $10 \%$ for men and $5 \%$ for women in recent studies..$^{1-5}$ Early clinical observations suggest that certain nephrolithiasis conditions (eg, staghorn calculi) and genetic diseases (eg, primary hyperoxaluria; dental disease; 2,8-hydroxyadenine crystalluria; cystinuria) are leading to progressive loss of epidermal growth factor receptor (eGFR) and end-stage renal disease (ESRD) at a younger age. ${ }^{6}$ An increasing number of studies have been done over the past few years concerning the relationship between kidney stones (nephrolithiasis/urolithiasis) and the development of chronic kidney disease (CKD) ${ }^{1-7}$ Kidney stones were found to be a risk factor for CKD in a recent study by a group at the Mayo Clinic in Rochester, MN. ${ }^{3}$ Alexander et $\mathrm{al}^{7}$ found that even a single kidney stone episode was associated with a significant increase in the risk of adverse renal outcomes including ESRD, with median follow up of 11

Corresponding Author: Matthew D'Costa, MD, Department of Internal Medicine, Marshfield Clinic, 1000 N Oak Avenue, Marshfield,WI 54449, USA,

Tel: (7I5)387-5600 , Email: matthewdcosta@gmail.com years. A large prospective cohort study of people in the United Kingdom found that kidney stones posed a significant risk for ESRD in women, but not in men. They explained this difference by noting anatomical protection from obstructive complications due to differences in anatomy. ${ }^{5}$ Several studies have shown an association between nephrolithiasis and certain comorbidities. More recently, the association between nephrolithiasis and an increased risk of coronary artery disease in women was reported by Ferraro et al. ${ }^{8}$

Review of the literature involving rural Wisconsin and nephrolithiasis revealed one study by Penniston et $\mathrm{al}^{9}$ to determine the incidence, recurrence, and prevalence of kidney stones. They studied a population of approximately 85,000 individuals in the Marshfield Epidemiologic Study Area (MESA) from 1992 to 2008 and found 3,212 incident cases of kidney stones. They also verified the validity of the ICD-9

Received: December 19, 2014

Ist Revision: June 29, 2015

2nd Revision: May 2, 2016

Accepted: May 26, 2016 
codes 592.0 and 592.1 for identification of index cases. Unlike previously mentioned studies, this paper did not ascertain the relationship between kidney stones and CKD or the relationship of these entities and urologic procedures.

There are several potential mechanisms for kidney stones leading to CKD. Extension of calcifications of the renal interstitium and tubular basement membrane into the tubular lumen can lead to scarring and a decrease in renal function. ${ }^{7}$ Khan ${ }^{10}$ hypothesized that damage could be caused by reactive oxygen species in the setting of several diseases including diabetes, hypertension, hypercalciuria, hyperoxaluria, and hypocitraturia. Scarring and progressive loss of kidney function may also be caused by direct damage from crystallization of the tubular lumen, as well as recurrent obstructions from stones.

Kidney stones vary in their presentation and, thus, have a wide range of treatment modalities. Treatment is based on the size, type, and location of the stone. Most stones less than $5 \mathrm{~mm}$ can be treated conservatively with increased fluid intake, pain control as well as tamsulosin in some cases. Hydration is also continued after the insult to prevent further stone formation. In a review of multiple studies, Wang et $\mathrm{al}^{11}$ found that patients have decreased rates of recurrence when producing 3 to 4 liters of urine per day.

Certain stone types (struvite and staghorn) and larger stones require procedural intervention, as they are likely to cause progressive renal dysfunction if not removed. ${ }^{7}$ Stone removal was historically performed with open nephrolithotomy, but there has been a shift to endouroscopic procedures as well as extracorporeal shockwave lithotripsy (ESWL). ${ }^{6}$ However, there is a limited number of long-term studies evaluating the effects of these procedures on kidney function. Open lithotomy is associated with a positive trend towards increased risk in the development of CKD, but this is not statistically significant. ${ }^{12}$ It is known that ESWL can cause direct renal injury via vasoconstriction, ${ }^{6}$ but no studies have shown a statistically significant relationship between this relationship and the development of CKD. One long-term study showed that the risk for elevated serum creatinine levels is not increased in patients receiving conservative management, ESWL, or percutaneous nephrolithotomy. ${ }^{13}$

Regarding outcomes of urologic procedures, no evidence was found in the literature to suggest that ESWL results in long term deterioration of renal function in patients with chronic renal insufficiency or a solitary kidney. ${ }^{14}$ On the contrary, Yoo et $\mathrm{al}^{15}$ found, in a retrospective analysis of 131 nephrolithiasis patients with stage 3 and 4 CKD and mean observation period of 3.2 years, that stone removal by ESWL was associated with delayed deterioration of renal function in CKD patients with nephrolithiasis. Percutaneous renal surgery in the solitary kidney is safe, and it results in renal function preservation. Female gender and an immediate postoperative improvement in the modification of diet in renal disease eGFR are predictive of a sustained increase in that rate of renal preservation. ${ }^{16}$ El-Asemy et $\mathrm{al}^{17}$ did not find any significant effect on renal function or blood pressure at the one-year follow-up after ESWL in patients with a solitary kidney. Percutaneous nephrolithotomy, ESWL, or the two techniques combined were found to be equally efficacious for preserving renal function in patients with solitary kidney. ${ }^{18}$

\section{Methods}

Study Design

This study was approved by the Marshfield Clinic Institutional Review Board. We conducted a population-based, retrospective cohort study, with data abstracted from comprehensive electronic medical records in 24 zip codes in Wisconsin in MESA. There were 156,211 unique individuals in MESA Central and MESA North during the period from July 1991 to the end of December of 2007; we extracted data on patients with urolithiasis (stone formers). The risk of development of adverse renal outcomes was evaluated related to urolithiasis and urologic procedures.

\section{Study Population}

During the study period, 1,340 patients who were diagnosed with urolithiasis met inclusion criteria, and their data extracted from MESA. Among the 1,340 subjects, 446 (33.28\%) had urologic procedures. Urologic procedures were extracted by Current Procedural Terminology (CPT) codes (see Appendix, Table A1). The risks of development of CKD, elevated serum creatinine, and any-cause mortality were compared among patients that underwent procedures to those that did not.

\section{Patient Selection}

Patients were selected by International Classification of Disease, Ninth Revision (ICD-9) codes (592.0 and 592.1). Death certificates, serum creatinine levels, estimated glomerular filtration rate (eGFR), and urologic procedures were captured. The accuracy of data from MESA has been validated in previous reports to include $90 \%$ or greater of patient events. ${ }^{19}$

\section{Inclusion and Exclusion Criteria}

Patients with nephrolithiasis (stone formers) in the MESA database during the study period were included in the study. Exclusion criteria included: (1) patients with clinical CKD or end-stage renal disease (ESRD) before the index date or within 90 days after the index date, or when they had an elevated mean serum creatinine during the 3 years to 1 month before the index date; (2) patients with no follow-up clinic visit within 90 days after the index date; and (3) patients without incident CKD were censored as of their last clinic visit or death or on December 31, 2011, whichever came first.

\section{Case Definition}

An incident nephrolithiasis case was determined to be the index date or first diagnosis of nephrolithiasis by ICD-9 code. Urological procedure codes were captured after the index date, and the occurrence of CKD was assessed after the index 
date. CKD was identified by several approaches, including: (a) a clinical diagnosis of CKD by ICD-9 (see Appendix, Table A2) and Hospital Adaptation of the International Classification of Diseases 8 (HICDA-8); (b) deaths overall were identified from records, and whether CKD was listed as a cause of death; (c) a composite endpoint was defined by ESRD (dialysis or transplantation) or death with $\mathrm{CKD} ;{ }^{8}$ and (d) by serum creatinine levels available from the MESA electronic medical records, classified as elevated serum creatinine level $\left(>97.5^{\text {th }}\right.$ percentile in regional kidney donors: $>1.3 \mathrm{mg} / \mathrm{dl}$ in men and $>1.1 \mathrm{mg} / \mathrm{dl}$ in women), which identified individuals with an eGFR less than expected with normal aging (eGFR $<60 \mathrm{ml} / \mathrm{min}$ per $1.73 \mathrm{~m}^{2}$ using serum creatinine-based MDRD [Modification of Diet in Renal Disease] equation). To distinguish CKD from acute renal failure, we defined CKD as a sustained elevation in creatinine (and thus, a decreased GFR as defined above) for greater than 90 days. Our analyses required the first serum creatinine level greater than 90 days after the initial serum creatinine elevation (or eGFR $<60 \mathrm{ml} / \mathrm{min}$ per $1.73 \mathrm{~m}^{2}$ ) and all serum creatinine levels in between meet or exceed the threshold requirement.

\section{Covariates}

Diagnoses of tobacco use, alcohol use, hypertension, diabetes mellitus, obesity, dyslipidemia, gout, peripheral vascular disease, coagulation disorder, coronary artery disease, congestive heart failure, and cerebrovascular incident were identified by ICD-9 and HICDA-8 codes (see Appendix, Table A3).

\section{Statistical Analysis}

Chi-square test was used to assess the differences in gender and comorbidities at baseline, while Wilcoxon's rank sum test was conducted to analyze the difference in age. The comorbidity propensity score was derived using a logistic regression modeling approach which included all comorbidity variables specified in Table 1. Then, each patient's status of propensity score was further defined using the median of the propensity score as a cutoff (above versus at or below the median) for the purpose of covariate adjustment in the proportional hazards regression modeling. Cox proportional hazards models (adjusted for age, gender, and comorbidities using comorbidity propensity score) were performed to evaluate the risk for incident CKD, elevated serum creatinine, and any-cause mortality. We used an interaction term between follow-up time and procedure status (with vs. without) to test for the proportional hazards assumption for the Cox regression. The assumption holds for our study.

\section{Results}

\section{Baseline Characteristics}

Median ages for patients with urolithiasis with and without procedure were 45.5 years and 45.9 years, respectively. No significant difference was found $(P=0.8839)$. In stone formers with procedure, $58.07 \%$ were male and $41.93 \%$ were female; in stone formers without procedure, $58.28 \%$ were male and $41.72 \%$ were female. No significant difference was found $(P=0.9427)$. The mean $\pm \mathrm{SD}$ (range) follow-up to last clinic visit or death was $9.80 \pm 5.00$ yrs. (0.26 to 20.31) in stone formers with procedure and $9.51 \pm 4.84 \mathrm{yrs}$. (0.25 to 19.63) in stone formers without procedure.

\section{Comorbidities}

Table 1 shows the baseline comorbidities of the patients with urolithiasis, and the groups compared were those with and without procedures. The most frequent comorbidity in both

Table 1. Baseline comorbidities in stone formers with and without procedure

\begin{tabular}{llll}
\hline Comorbidities at baseline & $\begin{array}{l}\text { Stone formers with } \\
\text { procedure prevalence (\%) }\end{array}$ & $\begin{array}{l}\text { Stone formers without } \\
\text { procedure prevalence (\%) }\end{array}$ & $\boldsymbol{P}^{*}$ Value $^{*}$ \\
\hline Hypertension & $218 / 446(48.9)$ & $391 / 894(43.7)$ & 0.0748 \\
Obesity & $213 / 446(47.8)$ & $320 / 894(35.8)$ & $<0.0001$ \\
Diabetes & $148 / 446(33.12)$ & $277 / 894(31.0)$ & 0.4149 \\
Dyslipidemia & $272 / 446(61.0)$ & $519 / 894(58.1)$ & 0.3036 \\
Gout & $26 / 446(5.8)$ & $43 / 894(4.8)$ & 0.4261 \\
Alcohol & $7 / 446(1.6)$ & $20 / 894(2.2)$ & 0.4124 \\
Tobacco use & $109 / 446(24.4)$ & $207 / 894(23.2)$ & 0.6015 \\
Coronary artery disease & $59 / 446(13.2)$ & $102 / 894(11.4)$ & 0.3344 \\
Cerebral infarct & $25 / 446(5.6)$ & $75 / 894(8.4)$ & 0.0676 \\
Heart failure & $31 / 446(6.9)$ & $63 / 894(7.1)$ & 0.9481 \\
Peripheral vascular disease & $53 / 446(11.9)$ & $82 / 894(9.2)$ & 0.1202 \\
Coagulation disorder & $8 / 446(1.8)$ & $9 / 894(1.0)$ & 0.2251 \\
\hline
\end{tabular}

${ }^{*} P$ value was derived from the Chi-square test. 
groups was dyslipidemia $(61.0 \%$ in procedure group and $58.0 \%$ in non-procedure group). Other frequent comorbidities in both groups were hypertension, obesity, and diabetes mellitus. Obesity was the only comorbidity with a statistically significant difference between stone formers with procedure compared to stone formers without procedure ( $47.8 \%$ in stone formers with procedure, and $35.8 \%$ in stone formers without procedure $[P<0.0001])$.

\section{Urologic Procedures}

Table A4 (Appendix) shows the list of all urologic procedures with corresponding CPT codes, and the number of patients in which they were performed. All patients underwent a procedure that included cystoscopy. The most frequent urologic procedure was cystoscopy with insertion of ureteral stent (387 of 446, $86.77 \%$ ). A total of 25 of $446(5.6 \%)$ patients underwent litholapaxy (lithotripsy with subsequent stone removal) or lithotripsy accompanied by cystoscopy.

\section{Hazard of CKD}

Table 2 shows that stone formers who had urologic procedures were significantly at increased risk for development of elevated serum creatinine (Hazard Ratio (HR) $[95 \% \mathrm{CI}]=$ 1.49 [1.19-1.85]), adjusted for age, gender, and comorbidity propensity score. Both CKD $(\mathrm{HR}[95 \% \mathrm{CI}]=1.08[0.77$ $1.49])$ and all-cause mortality (HR $[95 \% \mathrm{CI}]=1.13[0.78$ 1.64]) yielded a slight positive trend towards increase risk, but they were not statistically significant.

\section{Discussion}

In this retrospective study, the correlation between urologic procedures and progression of CKD, and the outcomes of stone formers were evaluated. Baseline characteristics of age, gender, and comorbidities in the study group (stone formers with urology procedure) were compared to the reference group (stone formers without procedures). Median age of patients with kidney stone and CKD is similar to recent reports in the literature. ${ }^{1-3,7}$ There was no statistically significant difference among them except obesity.Obesity has reached an epidemic stage, and an increase in the incidence of CKD and nephrolithiasis has been demonstrated in obese patients. ${ }^{1,20}$ It is associated with higher incidence of urinary tract infections, excessive intake of lithogenic substances (purine-rich, calcium, and oxalate), and low fluid intake. Orlistat and bariatric surgeries are associated with hyperoxaluria with increased risk for subsequent stone formation and oxalate nephropathy. ${ }^{20}$

Treatment of the morbidly obese is somewhat limited by their body habitus. Some tables used for ESWL have a maximum supported weight which can prohibit a morbidly obese patient from receiving that intervention. While there is a lack of literature in this area, increased subcutaneous tissue in the obese could theoretically provide an added obstacle for percutaneous nephrolithotomy. Regarding cystoscopy, one study of morbidly obese patients compared to normal weight individuals undergoing stone-matched procedures revealed no statistically significant difference in outcomes. ${ }^{21}$ It is unclear as to why our study revealed that stone formers undergoing procedures were more likely to be obese. It is possible they may be more likely to have stones requiring intervention although a review of the literature did not reveal studies where this would be the case. Detection bias may have also played a role since this variable is entirely dependent on physician documentation and whether or not weight, height and body mass index measurements were available at the time of kidney stone diagnosis and urologic procedure.

\section{Hazards among Stone Formers after Urologic Procedure}

We calculated the risk for elevated serum creatinine, CKD, and all-cause mortality in stone formers with and without procedures, with adjustment for age, gender, and baseline comorbidities using comorbidity propensity score. The vast majority of stone formers in our study had cystoscopy with stent placement (387 of 446), and as noted above, all patients that underwent a procedure that included cystoscopy.

We found that stone formers with procedures have a significantly increased risk of developing an elevated serum creatinine, which did not change substantively with adjustment for age, gender, and comorbidity propensity score. While this may be a result of procedural intervention, it is just as likely, if not more likely, to be a result of the renal injury secondary to the urolithiasis event itself. Patients that require procedural intervention are more likely to be at higher risk for a more significant renal injury from acute renal obstruction due to stone burden, size and type. The serial measurements of serum creatinine in these patients were theoretically used to monitor treatment response although we are unable to confirm this due to the retrospective nature of this study. Despite this significantly elevated creatinine, those that did receive procedures did not have a significantly higher incidence of CKD as the elevation in creatinine was not sustained for greater than ninety days. All-cause mortality was also not significantly increased in the procedure group. Not only do urologic procedures manage the kidney stone event itself, our study provides evidence that they may help to prevent long-term deterioration in kidney function as seen in previous studies. ${ }^{6,12-17}$

\section{Limitations}

There were several limitations to this study. Patients with kidney stones were identified either by seeking medical care for symptoms related to an acute episode or incidentally on imaging such as computed tomography or ultrasound. The former group would conceivably be more likely to have serum creatinine measurements at presentation and follow-up visits. This would lead to a possible detection bias. This method for identification of patients with kidney stone limits us from being able to study those that had asymptomatic kidney stones. Those who did not have a follow-up visit within 90 days after the index date were also excluded. It is likely that this occurred more often in patients who did not receive urological procedures, as the patients were seeking 
Table 2. Hazard ratios (HR) with $95 \%$ confidence intervals of clinical CKD, any-cause mortality, and elevated serum creatinine in stone formers with procedures compared to those without (reference group) with adjustment for age, gender, and baseline comorbidities using comorbidity propensity score.

\begin{tabular}{llll}
\hline & $\begin{array}{l}\text { Clinical CKD by any } \\
\text { diagnostic code }\end{array}$ & Any-Cause Mortality & $\begin{array}{l}\text { Elevated Serum } \\
\text { Creatinine }\end{array}$ \\
\hline Age, gender & $1.16(0.84-1.61)$ & $1.08(0.75-1.56)$ & $1.53(1.23-1.90)$ \\
$\begin{array}{l}\text { Age, gender, PS } \\
\text { ( } \leq \text { vs. }>\text { median) }\end{array}$ & $1.08(0.77-1.49)$ & $1.13(0.78-1.64)$ & $1.49(1.19-1.85)$ \\
\hline
\end{tabular}

PS, propensity score

this intervention likely due to complications related to the kidney stone event. Most often, patients will pass a stone and not seek further medical care. Given the limitations of data extraction based on this exclusion, we were unable to assess how these patients faired after the index event.

Regarding the patient composition, the MESA database is comprised mainly of Caucasian individuals, and prior studies ${ }^{5}$ have shown certain ethnic groups have different predispositions for kidney stone formation. While the MESA database is validated and patients mainly stay within the Marshfield Clinic healthcare system, there may be some kidney stone events, CKD diagnoses, and serum creatinine values not captured at healthcare facilities outside the MESA network. Thus, not all events are captured, and ICD-9 codes can be inaccurately recorded. Within this database, we were met with a low number of urologic procedures other than cystoscopy. This did not allow us to compare the risk among certain interventions for the development of CKD. Regarding comorbidities, liver disease is a major contributor to chronic kidney disease. Unfortunately we did not include diseases such as cirrhosis, portal hypertension, and chronic hepatitis infections in the initial analysis. Adjusting for these may provide some additional insight into our patient population. In addition, we did not adjust for various conditions that increase the risk for kidney stones such as hyperparathyroidism.

Due to the limitations of a retrospective chart review, we were unable to obtain information about stone composition, size and number due to the fact that many stones are passed without collection and analysis. Because of the very small number of procedures other than cystoscopy with stent placement, we were unable to evaluate the impact of different procedures on the course after management of urolithiasis.

\section{Conclusion}

This study revealed that stone formers who underwent procedures were at significantly increased risk for developing elevated serum creatinine, but were not at significantly increased risk for CKD and any-cause mortality compared to those stone formers without procedures. Urologic procedures may protect those with more significant urolithiasis events from long-term renal dysfunction. Further studies with larger sample sizes will be needed to determine if various procedures differ in preservation of renal function.

\section{Acknowledgements}

Funding for this study was provided by the Marshfield Clinic Resident Research Funds. The authors acknowledge the statistical assistance of Dr. Hong Liang and Dr. Po-Huang Chyou of the Marshfield Clinic Research Foundation's (MCRF) Bioinformatics Research Center. We also acknowledge the assistance of Sarah Kopitzke and Dr. Jeremy Pomeroy of MCRF's Center for Clinical Epidemiology \& Population Health. Finally, we thank MCRF's Office of Scientific Writing and Publication for editorial assistance in the preparation of this report.

\section{References}

1. Saucier NA, Sinha MK, Liang KV, et al. Risk factors for CKD in persons with kidney stones: a case-control study in Olmsted County, Minnesota. Am J Kidney Dis. 2010;55(1):61-68.

2. Worcester EM, Parks JH, Evan AP, Coe FL. Renal function in patients with nephrolithiasis. J Urol. 2006;176(2):600-603, discussion 603.

3. Rule AD, Krambeck AE, Lieske JC. Chronic kidney disease in kidney stone formers. Clin J Am Soc Nephrol. 2011;6(8): 2069-2075.

4. Stankus N, Hammes M, Gillen D, Worcester E. African American ESRD patients have a high pre-dialysis prevalence of kidney stones compared to NHANES III. Urol Res. 2007;35(2):83-87.

5. Hippisley-Cox J, Coupland C. Predicting the risk of Chronic Kidney Disease in Men and Women in England and Wales: prospective derivation and external validation of the QKidney® Scores. BMC Fam Pract. 2010;11(1):49.

6. Rule AD, Bergstralh EJ, Melton LJ III, Li X, Weaver AL, Lieske JC. Kidney stones and the risk for chronic kidney disease. Clin J Am Soc Nephrol. 2009;4(4):804-811.

7. Alexander RT, Hemmelgarn BR, Wiebe N, et al; Alberta Kidney Disease Network. Kidney stones and kidney function loss: a cohort study. BMJ. 2012;345(aug29 2):e5287.

8. Ferraro PM, Taylor EN, Eisner BH, et al. History of kidney stones and the risk of coronary heart disease. JAMA. 2013;310(4):408-415.

9. Penniston K, McLaren ID, Greenlee RT, Nakada SY. Urolithiasis in a Rural Wisconsin Population from 1992 to 2008: Narrowing of the Male-To-Female Ratio. J. Urology 2011;185(5):1731-1736.

10. Khan SR. Stress oxidative: nephrolithiasis and chronic kidney diseases. Minerva Med. 2013;104(1):23-30

11. Wang CJ, Grantham JJ, Wetmore JB. The medicinal use of water in renal disease. Kidney Int. 2013;84(1):45-53.

12. Eassa WA, Sheir KZ, Gad HM, Dawaba ME, El-Kenawy MR, Elkappany HA. Prospective study of the long-term effects of shock wave lithotripsy on renal function and blood pressure. $\mathrm{J}$ Urol. 2008;179(3):964-969, discussion 968-969. 
13. Krambeck AE, LeRoy AJ, Patterson DE, Gettman MT. Long-term outcomes of percutaneous nephrolithotomy compared to shock wave lithotripsy and conservative management. J Urol. 2008;179(6):2233-2237.

14. Chandhoke PS, Albala DM, Clayman RV. Long-term comparison of renal function in patients with solitary kidneys and/or moderate renal insufficiency undergoing extracorporeal shock wave lithotripsy or percutaneous nephrolithotomy. J Urol. 1992;147(5):1226-1230.

15. Yoo DE, Han SH, Oh HJ, et al. Removal of kidney stones by extracorporeal shock wave lithotripsy is associated with delayed progression of chronic kidney disease. Yonsei Med J. 2012;53(4):708-714.

16. Canes D, Hegarty NJ, Kamoi K, et al. Functional outcomes following percutaneous surgery in the solitary kidney. J Urol. 2009;181(1):154-160.

17. El-Assmy A, El-Nahas AR, Hekal IA, Badran M, Youssef RF, Sheir KZ. Long-term effects of extracorporeal shock wave lithotripsy on renal function: our experience with 156 patients with solitary kidney. J Urol. 2008;179(6):2229-2232.

18. Liou LS, Streem SB. Long-term renal functional effects of shock wave lithotripsy, percutaneous nephrolithotomy and combination therapy: a comparative study of patients with solitary kidney. J Urol. 2001;166(1):33-37, discussion 36-37.

19. Greenlee RT. Measuring Disease Frequency in the Marshfield Epidemiologic Study Area (MESA). Clin Med Res. 2003;1(4):273-280.

20. Ahmed MH, Ahmed HT, Khalil AA. Renal stone disease and obesity: what is important for urologists and nephrologists? Ren Fail. 2012;34(10):1348-1354.

21. Dash A, Schuster TG, Hollenbeck BK, Faerber GJ, Wolf JS Jr. Ureteroscopic treatment of renal calculi in morbidly obese patients: a stone-matched comparison. Urology. 2002;60(3):393-397, discussion 397.

\section{Author Affiliations}

Matthew D'Costa, MD*; Radmila Savcic-Kos, $M D^{*}$; Jingbo

Huang, $M D^{+}$; Andrew D. Rule, $M D^{\S}$; Narayana Murali, $M D^{\uparrow}$

*Department of Internal Medicine, Marshfield Clinic,

Marshfield, Wisconsin, USA

Department of Nephrology, Summit Ridge Medical Center,

Reno, Nevada, USA

*Division of Nephrology, UCLA-David Geffen School of

Medicine, Los Angeles, California, USA

$\S$ Division of Nephrology and Hypertension, Mayo Clinic,

Rochester, Minnesota, USA

"Department of Nephrology, Marshfield Clinic, Marshfield,

Wisconsin, USA 


\section{Appendix:}

Table A1. Urologic procedures with Current Procedural Terminology codes

\begin{tabular}{ll}
\hline CPT code & Procedure \\
\hline 52317 & Litholapaxy simple/sm $<2.5 \mathrm{~cm}$ \\
52318 & Litholapaxy simple/sm $>2.5 \mathrm{~cm}$ \\
52325 & Cystoscopy, fragmentation ureteral stone \\
52332 & Cystoscopy w/insertion ureteral stent \\
52335 & Endoscopy of urinary tract \\
52336 & Cystoscopy, stone removal \\
52337 & Cystoscopy, stone removal \\
52340 & Cystourethroscopy w/incision, fulgeration, resecection valves \\
52344 & Cystoscopy w/ureteroscopy w/treatment ureteral stricture \\
52351 & Cystoscopy w/ueretroscopy and pyeloscopy, diagnostic \\
52353 & Cystoscopy w/ureteroscopy w/lithotripsy \\
\hline
\end{tabular}

Table A2. Clinical diagnosis of CKD with corresponding ICD-9 codes

\begin{tabular}{ll}
\hline Clinical CKD & ICD-9 Codes \\
\hline Diabetes with renal manifestations & 250.4 \\
Gouty nephropathy & $274.10,274.19$ \\
Hypertensive renal disease & 403 \\
Hypertensive heart and renal disease & 404 \\
Goodpasture syndrome & 446.21 \\
Renal vein thrombosis & 453.3 \\
Hepatorenal syndrome & 572.4 \\
Nephrotic syndrome & 581 \\
Chronic glomerulonephritis & 582 \\
Nephritis and nephropathy & 583 \\
Chronic renal failure & 585 \\
Unspecified renal failure & 586 \\
Renal sclerosis & 587 \\
Disorder of kidney and ureter & 593.89 \\
Unspecified disorder of kidney and ureter & 593.9 \\
Cystic kidney disease & 753.1 \\
Renal agenesis & 753.0 \\
Congenital kidney anomalies & 753.3 \\
Proteinuria & 791.0 \\
\hline
\end{tabular}

CKD, chronic kidney disease 
Table A3. Comorbidities with corresponding ICD-9 codes

\begin{tabular}{ll}
\hline Comorbidity & ICD-9 Codes \\
\hline Obesity & $278.0,278.1,278.8$ \\
Dyslipidemia & $272.0,272.1,272.2,272.3,272.4$ \\
Alcohol Dependence & $291,303,305, \mathrm{~V} 79.1$ \\
Tobacco Use & $305.1,989.84, \mathrm{~V} 15.82$ \\
Diabetes & $250,357.2,362.01,362.02$, \\
Hypertension & $366.41,648.8,790.2$ \\
Coagulation defects & 401 \\
Gout & 286 \\
Coronary artery disease & $274.0,274.81,274.82,274.89$, \\
Heart failure & 274.9 \\
Cerebral infarct & $410,411,412,413$ \\
& $402.91,428.0,428.1,428.9$ \\
& $430,431,432.9,433,434,435.0$, \\
Peripheral vascular disease & $435.1,435.8,435.9,436,437.0$, \\
& $437.1,437.5,437.9$ \\
& $557.1,557.9,593.81$
\end{tabular}

Table A4. All urology procedures with CPT codes and number of patients in which they were performed.

\begin{tabular}{llc}
\hline CPT code & \multicolumn{1}{c}{ Procedure } & Number \\
\hline 52317 & Litholapaxy simple/sm <2.5 cm & 8 \\
52318 & Litholapaxy simple/sm $>2.5 \mathrm{~cm}$ & 6 \\
52325 & Cystoscopy, fragmentation ureteral stone & 1 \\
52332 & Cystoscopy w/insertion ureteral stent & 387 \\
52335 & Endoscopy of urinary tract & 2 \\
52336 & Cystoscopy, stone removal & 17 \\
52337 & Cystoscopy, stone removal & 4 \\
52340 & Cystourethroscopy w/incision, fulgeration, resecection valves & 1 \\
52344 & Cystoscopy w/ureteroscopy w/treatment ureteral stricture & 1 \\
52351 & Cystoscopy w/ueretroscopy and pyeloscopy, diagnostic & 8 \\
52353 & Cystoscopy w/ureteroscopy w/lithotripsy & 11 \\
\hline Total & & 446 \\
\hline CPT, Current Procedural Terminology & & 1 \\
\hline
\end{tabular}

